\title{
EDITORIAL
}

\section{Contemporary criteria for the selection of patients for percutaneous balloon mitral valvuloplasty}

\section{B D Prendergast, T R D Shaw, B lung, A Vahanian, D B Northridge}

Heart 2002;87:401-404

Percutaneous balloon mitral valvuloplasty is now the treatment of choice for many patients with symptomatic mitral stenosis Applications are expanding to include several categories of patients previously considered ineligible for the procedure.

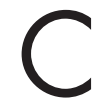
ommissural fusion is now recognised as the principal pathology underlying mitral stenosis, and commissural splitting underlies successful interventional treatment. Although the technique of surgical commissurotomy was first described as early as $1948,{ }^{1}$ percutaneous commissurotomy became a feasible option with the advent of the Inoue balloon in $1984 .{ }^{2}$ Percutaneous balloon mitral valvuloplasty (PBMV) is now the treatment of choice for many patients with symptomatic mitral stenosis. Numerous large series have reported excellent short, medium, and long term outcome ${ }^{3-7}$ with a low incidence of serious complications. ${ }^{8}$ Furthermore, randomised trials comparing balloon valvuloplasty with the surgical alternatives of open or closed commissurotomy have demonstrated equivalent outcome, ${ }^{9}{ }^{10}$ although patients treated using the percutaneous approach enjoy the advantages of reduced procedural morbidity and mortality and a short hospital stay. Specific advantages of the Inoue balloon in comparison with other percutaneous techniques include a lower risk of complications (particularly left ventricular perforation which is more frequent using double balloon techniques), easier manoeuvrability, its slender profile (creating a smaller defect in the interatrial septum), its self positioning characteristics, short inflation-deflation cycle, and capacity to permit gradually increasing successive balloon inflation sizes, which allow the operator to terminate the procedure when commissural splitting is achieved or when there is an increase in the severity of mitral regurgitation. Disadvantages include circumferential application of pressure during balloon inflation, occasionally resulting in paracommissural tears, especially in degenerate, calcified valves.

In developing nations, the application of PBMV is frequently limited on account of the high cost of the Inoue balloon. The development of a percutaneous metallic valvulotome, which can be autoclaved after each procedure to allow repeated use in many patients, may help to overcome these problems. Further potential advantages include a greater increase in valve area in comparison with the Inoue balloon, and selective commissural splitting, especially in calcified valves with a reduction in leaflet trauma. Initial data have been encouraging $^{11}{ }^{12}$ but we must await multicentre experience and the results of ongoing assessment in randomised controlled trials.

Given the success of PBMV, its applications are now expanding to include several categories of patients previously considered ineligible for the procedure. Nevertheless, the need for careful selection of appropriate patients remains paramount. In this article, we review the characteristics which need to be addressed and contemplate alternative approaches to be considered in certain circumstances.

\section{ECHOCARDIOGRAPHIC ASSESSMENT} Patients with non-pliable and/or calcified mitral valves

Initial experience of the procedure was confined to patients with pliable, non-calcified mitral valves. Indeed, early risk scores ${ }^{13}$ involving semiquantative assessment of leaflet thickening, subvalvar change, leaflet mobility, and valve calcification, emphasised the importance of these characteristics. However, these scores were largely derived from two dimensional transthoracic echocardiographic studies performed in younger subjects and their validity has been questioned. Although we have no randomised study available in the subgroup of patients with non-pliable calcified valves, it is now accepted that a favourable clinical and procedural outcome may be predicted in many subjects depending on the precise assessment of commissural morphology and a number of clinical and procedural variables.

\section{Commissural morphology}

In vitro studies indicate that the mechanism of successful PBMV is commissural splitting ${ }^{14}$ and several studies have emphasised the importance of commissural assessment in predicting outcome following the procedure. ${ }^{15-20}$ Commissural fusion may be assessed accurately using transthoracic (parasternal short axis view) and transoesophageal (mid-oesophageal transverse and horizontal plane) imaging, and calcification is indicated by the presence of localised bright echo reflections which cast an acoustic shadow across the commissure. In doubtful cases, precise localisation is facilitated by the improved resolution of transoesophageal imaging.

In one study, commissural calcium predicted reduced survival at 36 months free of the combined end point of death, repeat balloon valvotomy or need for mitral valve replacement. ${ }^{16}$ Regression analysis, which included conventional echocardiographic scores, identified commissural calcification as the only significant variable in the 
determination of outcome. The presence of commissural calcification may also predict major complications of PBMV. ${ }^{17}$

In a Parisian series of 422 patients with calcific mitral stenosis (assessed by means of a fluoroscopic score), a good immediate result following PBMV was obtained in 76\%. However, late deterioration was frequent during follow up, particularly following an initial suboptimal result. Multivariate analysis demonstrated the importance of a number of clinical and echocardiographic variables on initial and long term outcome, including the extent of calcification. ${ }^{18}$

Similarly, in a consecutive series of 300 Scottish patients, grade of commissural calcification (assessed by detailed transthoracic echocardiographic examination) was a significant predictor of successful outcome following PBMV. ${ }^{19}$ Furthermore, in a smaller series of 45 patients assessed prospectively by transoesophageal echocardiography, commissural calcification was the strongest independent predictor of an unsuccessful outcome following PBMV. Valves with minimal fusion were also unlikely to achieve a successful result. ${ }^{20}$

Clinical and procedural variables

Data from large series indicate that the prediction of outcome following PBMV is multifactorial and based not only on morphological characteristics of the valve but also a number of clinical and procedural variables, including age, functional class, effective balloon dilating area, and the final valve area. ${ }^{3421}$ Thus, balloon dilatation may well provide good long term results in patients with adverse anatomy if other characteristics are favourable. Conversely, if other characteristics are not in favour, then mitral surgery should be considered as an alternative.

A final factor influencing case selection is the experience of the clinical team. Several series have confirmed that complication rates are lower in high volume centres ${ }^{8}$ and, in our view, PBMV should be restricted to groups whose experience of transseptal catheterisation has been positive and who have been able to carry out an adequate number of procedures to maintain technical competence.

Overall, therefore, case selection is based on consideration of both clinical and morphological variables. Most operators now recommend percutaneous balloon valvotomy in patients with no commissural calcification despite a high overall echocardiographic score, particularly if there is echocardiographic evidence of commissural fusion and other clinical variables are favourable. Mitral valve replacement is the treatment of choice for patients with bicommissural calcification, and for most patients with calcification localised to one commissure. However, a percutaneous approach may still be considered in patients with commissural calcification who have an unacceptably high risk for heart surgery (for example, the frail elderly), especially when there is echocardiographic evidence of commissural fusion. In these difficult circumstances we emphasise the importance of overall clinical and echocardiographic assessment and the need for both physician and patient to be aware of the higher risk, the lower success rate, and suboptimal long term outcome. This palliative approach is generally supported by the use of smaller balloon inflations and ready acceptance of a suboptimal final valve area $\left(1.0-1.5 \mathrm{~cm}^{2}\right)$, especially when initial dilatation is associated with the development of mild, well tolerated mitral regurgitation.

\section{Patients with associated mitral regurgitation}

Overall, the incidence of severe mitral regurgitation complicating PBMV ranges from $2-19 \%$ in the major series. ${ }^{8}$ PBMV may be performed safely and successfully in patients with mitral stenosis and associated Sellers grade 1 mitral regurgitation. The stepwise dilatation technique reduces the risks of iatrogenic mitral regurgitation and also prevents a deterioration in pre-existent mitral regurgitation. Indeed, mild mitral regurgitation may improve or disappear following successful PBMV because of improved leaflet coaptation (A Vahanian, unpublished observations). The treatment decision is more controver- sial in patients with grade 2 mitral regurgitation, firstly since definition is difficult because of variability of measurement and, secondly, since clinical data are lacking. Once more, we recommend that the decision should be made in the light of other clinical and echocardiographic variables: surgery is the preferred option in a patient with unfavourable echocardiographic characteristics, whereas balloon dilatation is a better option in patients at high surgical risk. PBMV is not recommended in patients with Sellers grade 3 or 4 mitral regurgitation.

\section{Patients with left atrial thrombi}

The approach in these patients is controversial. Most specialists agree with the recently published American College of Cardiology/American Heart Association guidelines ${ }^{22}$ and avoid PBMV in the presence of left atrial thrombus which persists despite anticoagulant treatment, even if it is localised to the left atrial appendage. However, the low profile and manoeuvrability of the Inoue balloon catheter reduce the risk of dislodging a partially organised mural thrombus; in experienced hands, it is feasible to perform PBMV using a modified Inoue technique in selected patients with laminar thrombus confined to the left atrial appendage, ${ }^{23}$ particularly after 4-6 weeks of intense preprocedural treatment with warfarin.

\section{CLINICAL FEATURES \\ The elderly}

Most patients with symptomatic mitral stenosis in developed countries are elderly. Although degenerative changes affect commissural morphology, the mitral annulus, and subvalve apparatus in many of these patients, successful PBMV has been described in several series of elderly subjects. ${ }^{24}{ }^{25}$ Age alone is not a contraindication to the procedure. In fact, the indication may be extended to include palliation of high risk patients unsuitable for cardiac surgery whose valves are morphologically unsuitable for balloon dilatation.

\section{Patients with coexistent coronary artery disease}

Concomitant coronary artery disease with associated angina is a relatively frequent finding in the older population of patients with mitral stenosis in developed countries. In a consecutive series of 406 Scottish patients undergoing PBMV (mean (SD) age 61 (12) years), coronary artery disease was present in $21 \%$ (TRD Shaw, unpublished data, 2000). Successful PBMV followed by coronary angioplasty as a combined procedure is a feasible proposition in patients with mitral stenosis and single vessel coronary artery disease. ${ }^{26}$

\section{Patients with previous commissurotomy}

The incidence of restenosis after initially successful PBMV is around $40 \%$ after seven years. ${ }^{27}$ Overall event-free survival is inferior in patients undergoing repeat PBMV following previous surgical ${ }^{28}$ or balloon commissurotomy, ${ }^{29}$ since valves frequently develop further restenosis because of progressive degeneration and calcification of the leaflets and subvalve apparatus, recurrence of commissural fusion, or inadequate commissural splitting during the initial procedure. However, the immediate outcome and long term follow up results are excellent in carefully selected patients with suitable valves, and PBMV is a valuable treatment option in this group. ${ }^{29}$

\section{Pregnant patients with symptomatic mitral stenosis}

Data from about 100 pregnant patients indicate that PBMV is a safe and efficacious treatment to improve the mother's haemodynamic status; it is also well tolerated by the fetus. ${ }^{30}$ The risks of exposing the fetus to radiation may be minimised by adequate lead screening of the abdomen, and case reports describing a successful outcome using transoesophageal echocardiographic guidance alone (that is, dispensing with radiation) have recently appeared. It must be borne in mind, however, that the procedure always carries a risk of complications, albeit small. Therefore, it should be undertaken, when 
possible, before conception in women with severe mitral stenosis anticipating pregnancy in whom the development of symptoms seems likely, or limited to those patients-usually in the second trimester of pregnancy-who remain symptomatic despite appropriate medical treatment with diuretics and $\beta$ blockers.

\section{Patients with minimal symptoms}

PBMV may be considered in mildly symptomatic patients in the hope that early intervention will allow the patient normal or near normal exercise tolerance. ${ }^{31}$ The procedure may also be indicated after an embolic event or in patients whose only symptoms are caused by atrial arrhythmias. Indications in truly asymptomatic patients include the need for major noncardiac surgery, anticipated pregnancy (see above), and possibly subjects with an increased risk of embolism (for example, heavy left atrial spontaneous echo contrast).

\section{Patients with multivalvar stenoses}

In patients with severe mitral stenosis and mild to moderate aortic stenosis, PBMV may delay the need for double valve replacement. Similarly, in a patient with a previous aortic valve replacement and symptomatic mitral stenosis, PBMV may obviate the need for a second valve replacement. The use of PBMV in combination with concomitant aortic and/or tricuspid valvuloplasty has now also been reported. ${ }^{32}$

\section{Patients with contraindications to transseptal puncture or without inferior vena caval access}

In experienced hands, the risks of transseptal puncture are low. In occasional circumstances, the procedure may be unsuccessful or contraindicated, and alternative techniques using a transjugular venous or transarterial retrograde approach have been described. ${ }^{33}$ Hazards of the latter technique include the possibility of improper positioning of the guidewire, with associated risk of chordal rupture, and peripheral arterial damage caused by passage of the large valvuloplasty balloon catheter.

\section{CONCLUSIONS}

The indications for PBMV are expanding and the procedure is now feasible in many situations where it was previously felt to be contraindicated. Balloon valvuloplasty is contraindicated when mitral stenosis is accompanied by significant mitral regurgitation (> Sellers grade 2 ) or bilateral commissural calcification, or when a thrombus which is mobile or situated in a high risk position (on the atrial septum, protruding into the left atrial cavity, or obstructing the mitral valve orifice) presents a high likelihood of systemic embolism. Relative contraindications include the presence of thrombus which is localised to the left atrial appendage and unilateral commissural calcification. The decision to proceed in these situations remains controversial and depends on cumulative assessment of clinical and echocardiographic variables and the alternative risks presented by mitral valve surgery.

The short term advantages and excellent long term results of PBMV indicate that it is now the procedure of choice in patients with mitral stenosis whose valves are anatomically suitable. ${ }^{34}$ Use of the Inoue balloon is now widespread and this will remain the technique of choice for the foreseeable future. The place of emerging technology, notably the metallic valvulotome which may have specific advantages in developing nations, remains to be determined and is currently being addressed in prospective randomised trials.

\section{Authors' affiliations}

B D Prendergast, T R D Shaw, D B Northridge, Department of Cardiology, Western General Hospital, Edinburgh, UK B lung, A Vahanian, Service de Cardiologie, Hopital Bichat, Paris, France

\section{REFERENCES}

1 Bailey CP. The surgical treatment of mitral stenosis (mitral commissurotomy). Dis Chest 1949;15:377-93

2 Inoue K, Okawi T, Nakamura T, et al. Clinical application of transvenous mitral commissurotomy by a new balloon catheter. J Thorac Cardiovasc Surg 1984;87:394-402

3 lung B, Cormier B, Ducimetière $P$, et al. Immediate results of percutaneous mitral commissurotomy. A predictive model on a series of 1514 patients. Circulation 1996;94:2124-30.

4 lung B, Cormier B, Ducimetière $P$, et al. Functional results 5 years after successful percutaneous mitral commissurotomy in a series of 528 patients and analysis of predictive factors. J Am Coll Cardiol 1996;27:407-14

5 Palacios IF, Tuczu ME, Weyman AE, et al. Clinical follow-up of patients undergoing percutaneous mitral balloon valvotomy. Circulation 1995;91:671-6.

6 The National Heart, Lung and Blood Institute Balloon Valvuloplasty Registry Participants. Multicenter experience with balloon mitral commissurotomy: NHIBI balloon valvuloplasty registry report on immediate and 30-day follow-up results. Circulation 1992;85:448-61.

7 lung B, Garbarz E, Michaud P, et al. Late results of percutaneous mitral commissurotomy in a series of 1024 patients. Analysis of late clinical deterioration: frequency, anatomic findings, and predictive factors. Circulation 1999;99:3272-8.

8 Vahanian A. Percutaneous mitral commissurotomy. Eur Heart $\mathrm{J}$ 1996;17:1465-9.

9 Reyes VP, Soma Raju B, Wynne J, et al. Percutaneous balloon valvuloplasty compared with open surgical commissurotomy for mitral stenosis. N Engl J Med 1994;331:961-7.

10 Ben Farhat M. Ayari M, Maatouk F, et al. Percutaneous balloon versus surgical closed and open mitral commissurotomy. Seven-year follow-up results of randomized trial. Circulation 1998;97:245-50.

11 Cribier A, Rath PC, Letac B. Percutaneous mitral valvotomy with a metal dilator. Lancet 1997:349:1667-8.

12 Cribier A, Eltchaninoff $H$, Konig R, et al. Percutaneous mechanical mitral commissurotomy with a newly designed metallic valvulotome. Immediate results of the initial experience in 153 patients. Circulation 1999:99:793-9.

13 Wilkins GT, Weyman AE, Abascal VM, et al. Percutaneous balloon dilatation of the mitral valve: an analysis of echocardiographic variables related to outcome and the mechanism of dilatation. Br Heart J 1988:60:299-308.

14 Ribeira PA, Zaibag M, Rajendran V, et al. Mechanism of mitral valve area increase by in vitro single and double balloon mitral valvotomy. Am J Cardiol 1988;62:264-9.

15 Fatkin D, Roy P, Morgan JJ, et al. Percutaneous balloon mitral valvotomy with the Inove single-balloon catheter : commissural morphology as a determinant of outcome. J Am Coll Cardiol 1993;21:390-7.

16 Cannan CR, Nishimura RA, Reeder GS, et al. Echocardiographic assessment of commissural calcium: a simple predictor of outcome after percutaneous mitral balloon valvotomy. J Am Coll Cardiol 1997;29:175-80

17 Padial LR, Freitas N, Sagie A, et al. Echocardiography can predict which patients will develop severe mitral regurgitation after percutaneous mitral valvotomy. J Am Coll Cardiol 1996;27:1225-31.

18 lung B, Garbarz E, Doutrelant L, et al. Late results of percutaneous mitral commissurotomy for calcific mitral stenosis. Am J Cardiol 2000:85:1308-14

19 Sutaria N, Northridge DB, Shaw TRD. Significance of commissural calcification on outcome of mitral balloon valvotomy. Heart 2000:84:398-402.

20 Sutaria N, Shaw TRD, Prendergast BD, et al. Effect of commissural fusion on outcome of balloon mitral valvotomy: a transoesophageal study [abstract]. Heart 1998;80(suppl1):37

21 Hildick-Smith DJR, Taylor GJ, Shapiro LM. Inove balloon mitral valvuloplasty: long-term clinical and echocardiographic follow-up of a predominantly unfavourable population. Eur Heart J 2000;21:1690-7.

22 Bonow RO, Carabello B, de Leon AC Jr, et al. ACC/AHA guidelines for the management of patients with valvular heart disease: a report of the American College of Cardiology/American Heart Association task force on practice guidelines (committee on management of patients with valvular heart disease). J Am Coll Cardiol 1998;32:1486-588.

23 Hung J-S. Mitral stenosis with left atrial thrombi: Inoue balloon catheter technique. In: Cheng TO, ed. Percutaneous balloon valvuloplasty. New York: Igaku-Shoin, 1992:280-93.

24 Tuzcu EM, Block PC, Griffin BP, et al. Immediate and long-term outcome of percutaneous mitral valvotomy in patients 65 years and older. Circulation 1992;85:963-71.

25 Shaw TRD, Elder AT, Flapan AD, et al. Mitral balloon valvuloplasty for patients aged over 70 years: an alternative to surgical treatment. Age Ageing 1991;20:299-303.

26 Pomerantz RM, Diver DJ, Safian RD. Concurrent balloon valvuloplasty and coronary angioplasty. In: Cheng TO, ed. Percutaneous balloon valvuloplasty. New York: Igaku-Shoin, 1992:491-7.

27 Hernandez R, Banuelos C, Alfonso F, et al. Long-term clinical and echocardiographic follow-up after percutaneous mitral valvuloplasty with the Inove balloon. Circulation 1999;99:1580-6. 
28 Jang IK, Block PC, Newell JB, et al. Percutaneous mitral balloon valvotomy for recurrent mitral stenosis after surgical commissurotomy. Am J Cardiol 1995;75:601-5

29 lung B, Garbarz E, Michaud P, et al. Immediate and mid-term results of repeat percutaneous mitral commissurotomy for restenosis following earlier percutaneous mitral commissurotomy. Eur Heart $J$ 2000;21:1683-9.

30 lung B, Cormier B, Elias J, et al. Usefulness of percutaneous balloon commissurotomy for mitral stenosis during pregnancy. Am J Cardiol 1994;73:398-400.
31 lung B, Garbarz E, Michaud P, et al. What are the long-term results of percutaneous mitral commissurotomy in patients with no or few symptoms? [abstract]. Circulation 1998;98(supp I):I-352.

32 Cheng TO. Multivalve percutaneous balloon valvuloplasty. Cathet Cardiovasc Diagn 1989:16:109-12.

33 Stefanidis C, Stratos C, Pitsavos C, et al. Retrograde nontransseptal balloon mitral valvuloplasty: immediate results and long-term follow-up. Circulation 1992;85:1760-7.

34 Palacios IF. Farewell to surgical mitral commissurotomy for many patients. Circulation 1998;97:223-6.

\section{IMAGES IN CARDIOLOGY}

\section{Ruptured thoracic aneurysm in a 10 year old boy}

10 year old boy with no prior medical history suddenly developed A severe pain between the shoulder blades, dyspnoea, and cyanotomographic (CT) and three dimensional imaging reconstructed from spiral CT (Vitrea) revealed an aneurysmal dilatation of the ascending aorta with a diameter of $81 \mathrm{~mm}$, extending from the aortic valve to just proximal of origin of the left subclavian artery (right, white arrow). The right pleural space contained an amount of free blood. A huge mediastinal haematoma surrounding the ascending aorta, compressing both the heart and the lungs and displacing the oesophagus laterally, is seen. In addition, CT angiography revealed a contrast leak in the posterior wall just behind the cerebral vessels (below, black arrow). Furthermore, severe calcifications of the aneurysmal aortic wall were evident, indicating pre-existing aortic pathology.

Emergency surgery included replacement of the ascending aorta and the inferior part of the aortic arch by a $22 \mathrm{~mm}$ Vascutec prosthesis, leaving an island of autologous tissue containing the head vessels intact. The postoperative course was uneventful.

Pathological examination of the resected aneurysmal tissue showed severe scarring and degeneration of the aortic wall with dystrophic calcifications, indicating a systemic cause. Predisposing factors, including hereditary connective tissue disease (Marfan's syndrome, Ehlers-Danlos syndrome), congenital heart abnormalities, hyper-

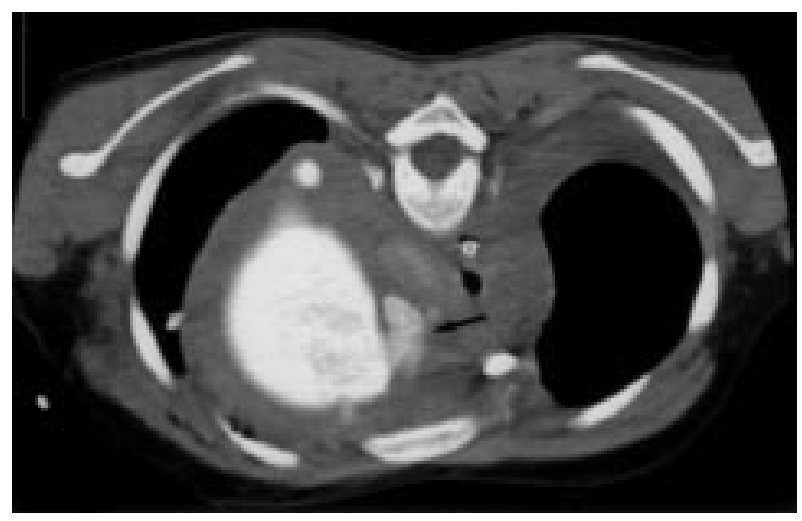

tension, trauma, infection (syphilis), and autoimmune disease, were excluded, making this case extremely rare.

M Noordzii

E Hanlo-Ploeger

E J Meijboom

e.meijboom@azu.nl

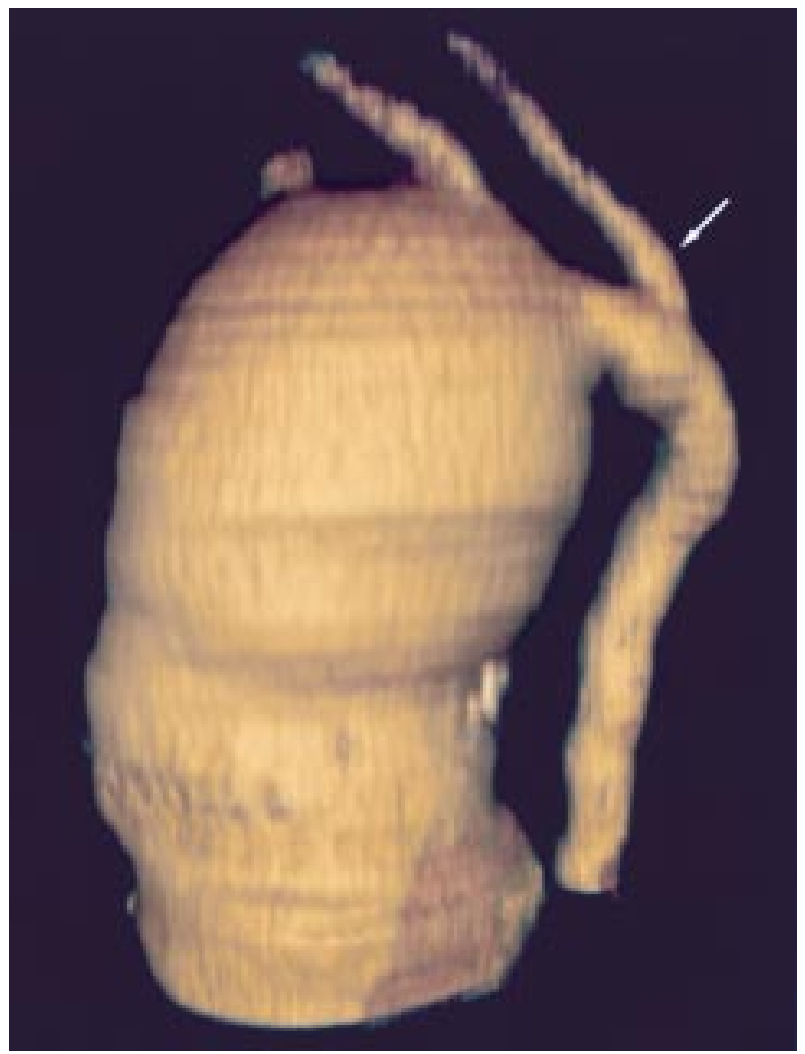

